\title{
Optical and Loss Spectra of Carbon Nanotubes: Depolarization Effects and Intertube Interactions
}

\author{
A. G. Marinopoulos, ${ }^{1}$ Lucia Reining, ${ }^{1}$ Angel Rubio, ${ }^{2}$ and Nathalie Vast ${ }^{1}$ \\ ${ }^{1}$ Laboratoire des Solides Irradiés, UMR 7642 CNRS/CEA, École Polytechnique, F-91128 Palaiseau, France \\ ${ }^{2}$ Departamento de Física de Materiales, Facultad de Químicas, Universidad del País Vasco, Centro Mixto CSIC-UPV/EHU \\ and Donostia International Physics Center (DIPC), 20018 San Sebastián/Donostia, Spain \\ (Received 29 October 2002; published 23 July 2003)
}

\begin{abstract}
We performed ab initio calculations of the anisotropic dielectric response of small-diameter singlewalled carbon nanotubes in the framework of time-dependent density-functional theory. The calculated optical spectra are in very good agreement with experiment, both concerning absolute peak positions and anisotropy effects. The latter can only be described correctly when crystal local-field effects ("depolarization" effects) are fully taken into account. Moreover, interactions between the tubes can strongly modify their absorption and electron energy-loss spectra.
\end{abstract}

DOI: 10.1103/PhysRevLett.91.046402

Research on carbon nanotubes [1] has been expanding rapidly owing to their unique properties, mechanical response, electrical transport, and conduction, being metallic or semiconducting depending on their diameter and the helicity of the rolled graphene layer. The electronic structure and dielectric properties of the tubes are two key areas to study. Important progress has been made in determining experimentally the electronic structure and core-level (carbon $K$ edge) spectra of isolated tubes by scanning tunneling spectroscopy [2] and spatially resolved electron energy-loss spectroscopy (EELS) [3], respectively. On the other hand, the dielectric response in the frequency range of the electronic interband transitions and the collective excitations (plasmons) of the valence electrons (up to $50 \mathrm{eV}$ ) is not well understood, and the existing calculations focused on the low-energy excitations (see, e.g., Ref. [4]). Therefore, important questions concerning the electron interaction, excitations, and screening still remain unanswered. Furthermore, the role of intertube interaction in the spectra needs to be addressed especially in solids and bundles where the individual tubes are close packed and could interact with each other via long-range forces induced by the excitations. Experimentally [5] it has not been possible to answer the above questions since in the bulk solid and even in bundles the tubes are not perfectly aligned and do not have a well-defined diameter and helicity, rendering a random mixture of semiconducting and metallic tubes. Only recently optical absorption spectra were reported [6] for aligned tubes of a very narrow diameter distribution $(4 \pm 0.2 \AA)$ allowing a direct comparison between experiment and theory. Since three possible tubular helicities exist for the range of diameters around $4 \AA$ - the armchair $(3,3)$, the zigzag $(5,0)$ and the chiral $(4,2)-a$ reliable theoretical approach for calculating spectra would have a significant role in the interpretation and analysis of the experimental results on given tubes concerning their helicity and interaction.
PACS numbers: 71.45.Gm, 77.22.Ej, 78.20.Bh, 78.67.Ch

Theoretical studies of the spectra of carbon tubes, besides model calculations [7], are mostly limited to summing over independent band-to-band transitions obtained within the tight-binding method [8] or the densityfunctional theory (DFT) framework [9,10], or to calculations of the joint density of states (JDOS) [11] which use the band structure with no explicit evaluation of the transition matrix elements. As we will show below, these approximations are not sufficient for a full interpretation of the experiments. This was evident in two recent DFTbased studies of the optical absorption spectra of the $4 \AA$ A-diameter tubes [9]. These calculations, although they successfully described the spectra for light polarized along the tube axis, could not explain the experimentally observed depolarization $[4,12]$ which takes place for perpendicular polarizations and renders the tubes transparent for frequencies below $4 \mathrm{eV}$ [6]. This shortcoming is not due to the quality of the band structure calculation itself but instead due to the neglect of the induced microscopic components in the response to the external field, the local-field effects (LFE), which strongly modify the results. Also, induced exchange and correlation (XC) components obtained beyond the random-phase approximation (RPA) might contribute $[4,13]$.

Today in the solid state $a b$ initio framework, two main approaches include these effects [13]: First, Green's functions approaches adding self-energy corrections to the DFT Kohn-Sham band structure and including the electron-hole interaction via the solution of the BetheSalpeter equation give excellent results but they are computationally too cumbersome to be applied systematically to complex systems. The time-dependent Hartree-Fock (TDHF) method can be regarded as an approximation to this approach, since it can be obtained from the latter simply by neglecting correlation (i.e., screening) in the self-energy, whereas LFE are still retained. It was used in a semiempirical way to calculate optical spectra, including excitons, of carbon nanotubes [4], also for the 
$4 \AA$-diameter ones [14]. The results in the latter case did not explain adequately the observed peaks and also were in contradiction with the peak assignments obtained from DFT calculations [6,9]. It is clear that in such systems with important metallic character a neglect of screening may lead to problems.

In this work we adopt a second approach, which is based on time-dependent DFT (TDDFT) [15]. LFE are formally correctly included. However, in practice XC are approximated; in particular, the adiabatic local density approximation (TDLDA) has been applied to many finite and infinite systems [13] and, of importance here (since the graphitic layers are the building block of the tubes), excellent results for the loss spectra of graphite were already obtained [16]. Here we report calculations of the absorption and loss spectra of the $4 \AA$ nanotubes in both RPA and the TDLDA.

We determined the ground state equilibrium geometry and Kohn-Sham band structure of parallel nanotubes arranged in a hexagonal lattice using $a b$ initio, normconserving pseudopotentials and a plane-wave basis [17]. The calculations were done for the $(3,3),(5,0)$, and $(4,2)$ tubes, and for an intertube distance (distance between tube walls), equal to $D_{t}=5.5 \AA$ which leads to nearly isolated tubes. For the $(3,3)$ armchair ones, we also performed calculations for a solid of interacting tubes with a distance $D_{t}=3.2 \AA$ close to the interlayer distance in graphite $(\sim 3.4 \AA)$. From this starting point, the absorption spectra were computed through the macroscopic dielectric function $\varepsilon_{M}(\omega)$ for a periodic system from

$$
\varepsilon_{M}(\omega)=1 / \varepsilon_{\mathbf{G}=\mathbf{G}^{\prime}=\mathbf{0}}^{-1}(\mathbf{q} \rightarrow \mathbf{0} ; \omega)
$$

( $\mathbf{q}$ is in the first Brillouin zone and $\mathbf{G}, \mathbf{G}^{\prime}$ are reciprocal lattice vectors). This is equivalent to calculating $\varepsilon_{M}=$ $1-V_{C} \bar{\chi}$, where $\bar{\chi}$ is a modified response function which obeys the matrix equation $\bar{\chi}=\chi_{0}+\chi_{0}\left(\bar{V}_{C}+f_{x c}\right) \bar{\chi}$ [13]. $\bar{V}_{C}(\mathbf{q}+\mathbf{G})$ is equal to the bare Coulomb interaction, $V_{C}$, but without its long-range $(G=0)$ contribution, $\chi_{0}$ is the independent-particle polarizability, and $f_{x c}$ is the $\mathrm{XC}$ kernel obtained as the functional derivative of the LDAXC potential with respect to the electron density [15]. Within RPA, $f_{x c}$ is assumed to be zero. Instead, $\bar{V}_{C}$ is the microscopic part of the density variation of the Hartree potential and corresponds to considering the off-diagonal elements of $\varepsilon$ in the matrix inversion in Eq. (1), i.e., to including LFE which are expected to be well described. When LFE are neglected, and moreover all transition matrix elements in $\chi_{0}$ are supposed to be constant, one arrives at the widely used approximation that the absorption spectrum is proportional to the JDOS, i.e., proportional to the sum over independent transitions from occupied $(v)$ to empty $(c)$ states over the Brillouin zone points $\mathbf{k}, \sum_{v, c, \mathbf{k}} \delta\left(\epsilon_{c \mathbf{k}}-\epsilon_{v \mathbf{k}}-\omega\right)$.

In Fig. 1 (upper panel), we show the JDOS divided by the square of the excitation energy, for the $(3,3)$ nearly isolated (thin line) and interacting (in the solid) (thick

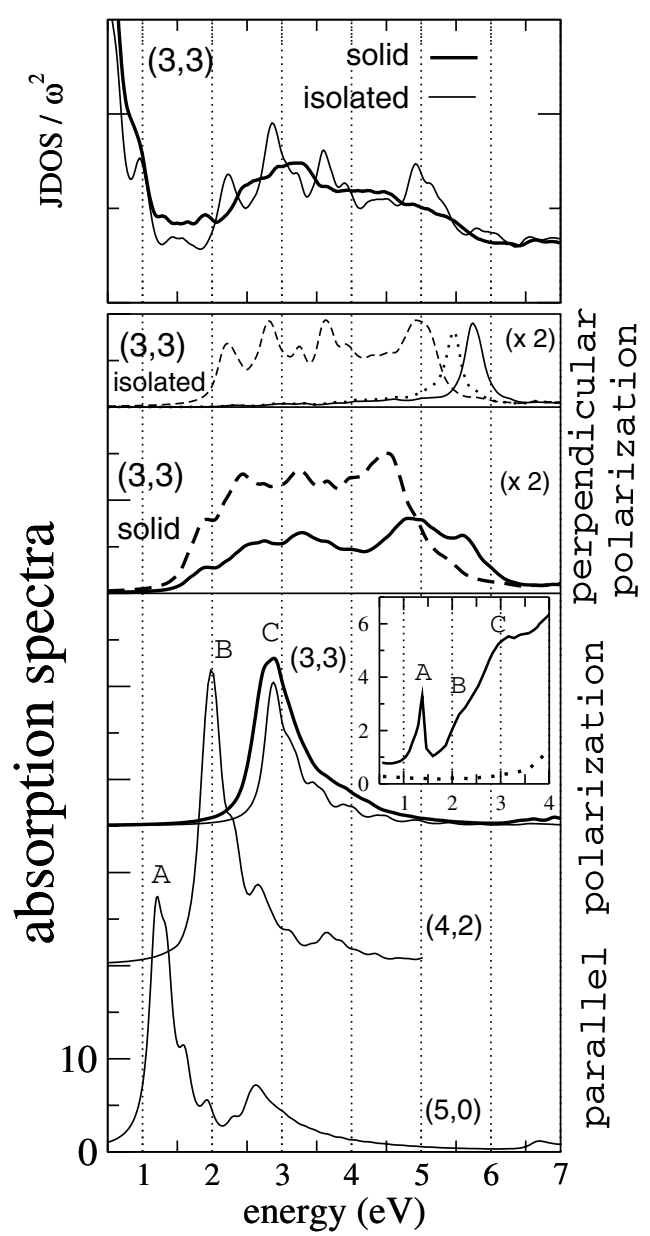

FIG. 1. Calculated RPA absorption spectra with (continuous lines) and without (dashed lines) LFE. For the (3,3) tubes, results for both the isolated ones (thin lines) and in the solid (thick lines) are presented. For comparison, the JDOS are also shown (upper panel). The TDLDA result for the $(3,3)$ isolated tubes is given in the second panel (dotted line). The results for the $(5,0)$ and $(4,2)$ tubes are only shown for the parallel polarization where LFE are negligible. The spectra for the perpendicular polarization have been multiplied by 2 . The experimental data [6] are shown in the inset for parallel (solid line) and perpendicular polarization (dotted line).

line) tubes. After an initial $(<1 \mathrm{eV})$ steep decrease, the two JDOS curves show an increase in the oscillator strength starting from $2 \mathrm{eV}$. For the isolated case, one sees a sequence of pronounced peak structures up to $5 \mathrm{eV}$ which can be explained from the occurrence of direct interband transitions between the Van Hove singularities of the density of states (DOS). These peaks are smeared out in the JDOS of the solid where the tubes are strongly interacting [18]. The lower panels of Fig. 1 show the calculated spectra for light polarization perpendicular and parallel to the tube axis. Dashed lines are results in the RPA neglecting LFE, continuous lines including LFE. For parallel polarization, only the RPA results without LFE are presented because in this case both LFE and XC effects turned out to be negligible. This explains the 
coincidence between the peak positions predicted here (A, B, and C in Fig. 1) with those obtained for this polarization in recent DFT-RPA calculations [9], where these effects were neglected and, therefore, justifies such a neglect for the parallel polarization. It is, hence, confirmed that one can predict the major peak structures of the experimental absorption spectrum (peaks A, B, and C in the inset of Fig. 1) with a good agreement (to within $0.2 \mathrm{eV}$ for our results) allowing the assignment of the peaks to specific tubular helicities. The effect of intertube interaction, instead, is small for this polarization and consists of the broadening of the main absorption peak [thick solid curve for the $(3,3)$ tubes]. This can be explained from an increase of the energy range of the possible interband transitions due to the interaction.

When LFE are neglected, $\operatorname{Im}\left(\varepsilon_{M}\right)$ is proportional to $\operatorname{Im}\left(\chi_{0}\right)$, and, hence, with respect to the JDOS, includes correctly transition matrix elements leading to a modification of intensities; it can, however, be seen that there is a strong resemblance and one-to-one correspondence between peaks in the weighted JDOS and in the spectra without LFE of the isolated $(3,3)$ tubes for all major peak structures (both polarizations together). All these findings seem to indicate that a JDOS, and, hence, finally a band structure calculation, is enough to understand the spectra. However, for perpendicular polarization the experimental absorption spectrum has vanishing intensity in the frequency range up to $4 \mathrm{eV}$ [6] (see inset of Fig. 1, dotted curve). Neither the JDOS calculation nor the calculated absorption spectrum without LFE can capture this effect. The reason is that a depolarization effect $[4,12]$ takes place originating from the presence of microscopic electric fields due to the induced polarization charges after the application of the external field. These depolarization fields are only accounted for if LFE are included in the calculations. In Fig. 1 (second panel; continuous curve) it is, indeed, seen that LFE suppress the absorption peaks and render the tubes almost transparent below $5 \mathrm{eV}$ in agreement with experiment. In contrast, the recent DFT-RPA calculations [9] were unable to reproduce this transparency since LFE were neglected.

The TDLDA result (dotted curve; second panel) is similar to the RPA-with-LFE result: In both cases the low-energy absorption peaks are suppressed. This shows that the main effect comes from fluctuations of the Hartree, and not from those of the XC potential. LDA$\mathrm{XC}$ effects only cause a small $(0.3 \mathrm{eV})$ redshift of the remaining absorption peak to $5.5 \mathrm{eV}$, with respect to RPA with LFE. This is a characteristic behavior of finite systems [13].

A similar impact of the LFE is found for the system of isolated $(5,0)$ tubes (not shown). This drastic effect can be understood from the fact that for perpendicular polarization the tubes form a system of almost isolated, but highly polarizable, objects. An external field induces, hence, a local, i.e., microscopic, response - the LFE - which strongly weakens the total perturbation (i.e., it is a depo- larization). The macroscopic response to this weak perturbation is only very moderate, because the electrons are localized on the tubes. This is totally different from a bulk metal or small-gap semiconductor, where even a very weak total perturbation still leads to a strong response at low frequencies. Parallel to the tube axis, the situation resembles in fact rather this latter case, which explains the absence of LFE for this polarization. The depolarization (for the perpendicular polarization) is much weaker when the tubes are interacting (third panel) making them no longer transparent, because the electronic states start to delocalize and the system is now more similar to a bulk metal. This has a very important consequence: It suggests that the intertube interaction can be detected experimentally in a qualitative study of the absorption spectra for perpendicular polarizations.

The intertube interaction has also an important effect on the loss function, notably in the range $15-35 \mathrm{eV}$ of the higher-frequency $\pi+\sigma$ plasmon. We stress here that, contrary to the absorption, the small- $q$ loss function contains the full long-range Coulomb interaction [13]; therefore the tubes cannot be considered as completely isolated objects in this calculation of the loss function with the $5.5 \AA$ intertube distance. We refer to the tubes in the latter geometry as distant. Figure 2 shows the RPA loss function, $-\operatorname{Im}\left(1 / \varepsilon_{M}\right)$, for the $(3,3)$ tube, for a vanishing momentum transfer $\mathbf{q}$ parallel to the tube axis. For this orientation LFE are negligible. A strong shift of the $\pi+\sigma$ plasmon from 22 to $28 \mathrm{eV}$ due to intertube interactions in the solid can be seen [19]. The magnitude of this shift indicates a strong dependence of the plasmon frequency upon the intertube distance (hence, the average valence electron density) essentially following the plasmon-frequency dependence in the case of the homogeneous electron gas [20]. This shows that actually in the long-wavelength limit $(\mathbf{q} \rightarrow \mathbf{0})$ and for parallel $\mathbf{q}$ orientations the tubes can be considered as homogeneous and highly polarizable media. Therefore the local atomic arrangement and helicities must play a secondary role and the present results for the $\pi+\sigma$ plasmon should be

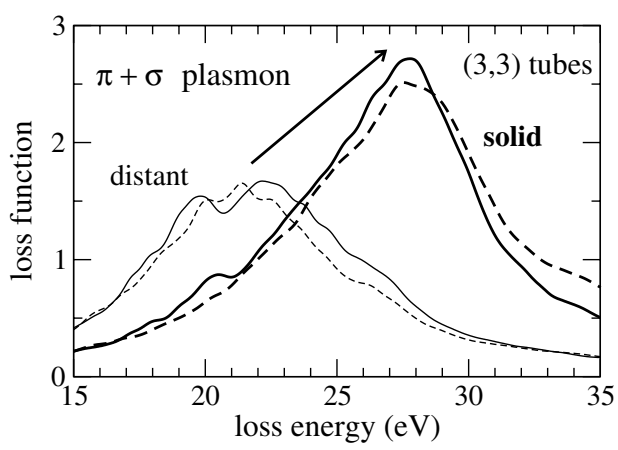

FIG. 2. Calculated RPA loss function for the (3,3) distant tubes (thin line) and solid (thick line) of tubes without LFE. q is parallel to the tube axis. Dashed curves are the results for graphite (thick) and graphene (thin) for in-layer q [16]. 
representative for either of the three tubes since all of them (being of nearly the same diameter) possess the same average electron density. Consequently, we argue that, contrary to an optical absorption experiment, a small- $q$ loss experiment measuring the $\pi+\sigma$ plasmon cannot determine tubular helicities for a given tube diameter. Our assertion is further supported by the fact that an almost indistinguishable $\pi+\sigma$ plasmon was also obtained for the $(5,0)$ tube (not shown) and (for in-layer q orientation) in graphite at comparable average electron densities (to within 10\%) [16] (dashed curves in Fig. 2). This shows that the loss function of the tubes for parallel $\mathbf{q}$ in this frequency range is governed by the averagedensity-dependent part of the in-layer graphitic response. Similar shifts, therefore, can also be expected in other graphite-based structures, e.g., multiwalled tubes. The present results outline the significance of the $\pi+\sigma$ plasmon as a key measurable spectroscopic quantity which could gauge the intertube distances and interactions in real samples.

Before concluding, it is important to address the validity of RPA and TDLDA for the description of the optical spectra of nanotubes. In fact, RPA and TDLDA often give excellent results for loss spectra [13] including those of graphite [16]. The present work, however, shows good RPA absorption spectra for the carbon tubes despite the fact that both RPA and TDLDA are known to fail badly in the description of absorption spectra of many bulk materials [13]. This seeming contradiction can be explained as follows: Parallel to the tubes, the screening is large. Therefore, XC effects are damped. Perpendicular to the axis, the distant tubes behave essentially like isolated systems, where strong cancellations are known to occur between self-energy corrections and electron-hole interaction (i.e., between XC) effects [13]. The experimental precision is then not high enough (also due to the depolarization effect) to discern to which extent $\mathrm{XC}$ effects should be better described, beyond TDLDA.

In conclusion, we performed the first ab initio RPA with LFE and TDLDA calculations of optical and energy-loss spectra of carbon nanotubes, obtaining a very good agreement with the existing absorption experiment. Our findings are relevant for understanding the dielectric response of other nanostructured and low-dimensional systems. We showed that the JDOS and the RPA neglecting LFE are not sufficient to fully interpret the experiment: LFE are crucial in order to explain the depolarization effect and the suppression of absorption peaks which occurs for the perpendicular polarization. The intertube interaction strongly weakens the depolarization and also determines the position of the $\pi+\sigma$ plasmon in the loss function. The position and also line shape of this plasmon in the interacting and isolated tubes for small and parallel $\mathbf{q}$ are very similar to those of graphite and graphene even for the small-diameter size studied here.

This work was supported by the EC-RTN program NANOPHASE (Contract No. HPRN-CT-2000-00167).
A. R. acknowledges support from the Ecole Polytechnique during a sabbatical leave and MCyT (MAT20010946). Computer time was granted by IDRIS (Project No. 544).

[1] See Carbon Nanotubes: Synthesis, Structure, Properties, and Applications, edited by M. S. Dresselhaus, G. Dresselhaus, and Ph. Avouris (Springer-Verlag, Berlin, 2001); R. H. Baugman, A. A. Zakhidov, and W. A. de Heer, Science 297, 787 (2002), and references therein.

[2] For example, J.W. G. Wildöer et al. Nature (London) 391, 59 (1998); T.W. Odom et al. Nature (London) 391, 62 (1998).

[3] See, e.g., O. Stéphan et al., J. Electron Spectrosc. Relat. Phenom. 114-116, 209 (2001).

[4] H. Ajiki and T. Ando, Physica (Amsterdam) 201B, 349 (1994); T. Ando, J. Phys. Soc. Jpn. 66, 1066 (1997).

[5] For example, W. A. de Heer et al., Science 268, 845 (1995); T. Pichler et al. Phys. Rev. Lett. 80, 4729 (1998); H. Kataura et al. Synth. Met. 103, 2555 (1999); X. Liu et al. Phys. Rev. B 66, 045411 (2002).

[6] Z. M. Li et al., Phys. Rev. Lett. 87, 127401 (2001).

[7] For example, C. Yannouleas, E. N. Bogachek, and U. Landman, Phys. Rev. B 53, 10225 (1996); F. J. Garcia-Vidal, J. M. Pitarke, and J. B. Pendry, Phys. Rev. Lett. 78, 4289 (1997).

[8] M. F. Lin and K.W. K. Shung, Phys. Rev. B 50, 17744 (1994); S. Tasaki et al., ibid. 57, 9301 (1998); M. F. Lin et al., ibid. 61, 14114 (2000); F. L. Shyu and M. F. Lin, ibid. 62, 8508 (2000).

[9] H. J. Liu and C. T. Chan, Phys. Rev. B 66, 115416 (2002); M. Machon et al., Phys. Rev. B 66, 155410 (2002).

[10] J.W. Mintmire and C.T. White, Synth. Met. 77, 231 (1996).

[11] I. Bozovic, N. Bozovic, and M. Damnjanovic, Phys. Rev. B 62, 6971 (2000); M. Ichida et al., ibid. 65, 241407 (2002); L. Wirtz et al., ibid. (to be published).

[12] L. X. Benedict, S. G. Louie, and M. L. Cohen, Phys. Rev. B 52, 8541 (1995).

[13] G. Onida, L. Reining, and A. Rubio, Rev. Mod. Phys. 74, 601 (2002).

[14] W. Z. Liang et al., Appl. Phys. Lett. 80, 3415 (2002).

[15] E. Runge and E. K. U. Gross, Phys. Rev. Lett. 52, 997 (1984); E. K. U. Gross, F. J. Dobson, and M. Petersilka, Density Functional Theory (Springer, New York, 1996).

[16] A. G. Marinopoulos et al., Phys. Rev. Lett. 89, 076402 (2002).

[17] M. C. Payne et al., Rev. Mod. Phys. 64, 1045 (1992). The pseudopotential has been generated following N. Troullier and J. L. Martins, Phys. Rev. B 43, 1993 (1991).

[18] See also, e.g., A. Rubio, Appl. Phys. A 68, 275 (1999).

[19] A similar effect was also seen in model calculations: G. Gumbs and G. R. Aizin, Phys. Rev. B 65, 195407 (2002).

[20] See, e.g., W. Jones and N. H. March, Theoretical Solid State Physics: Vol. 1 (Wiley-Interscience, New York, 1973). 